\title{
Stellar Zeeman Analyses: Effects of Multi-component Atmospheres
}

\author{
G.W. Marcy, G. Basri, J.A. Valenti \\ Astronomy Department, Univ. of California, Berkeley, CA 94720, USA
}

\begin{abstract}
We simulate the radiative transfer of Zeeman-broadened lines in an active star containing both quiet and flux-tube regions. A traditional one-atmosphere Zeeman analysis of these synthetic lines yields magnetic field measurements systematically inaccurate by up to $40 \%$. However, two atmospheric components alone cannot reproduce the observed line strengths, luminosities or photometric constancy of active stars.
\end{abstract}

\section{Introduction}

Previous magnetic analyses of cool dwarfs (e.g., Saar, 1988; Marcy and Basri, 1989 ) involve fitting observed Zeeman-broadened line profiles with synthetic profiles computed by assuming that the stellar atmosphere has only one component, thus ignoring the different depth structures of flux-tubes, spots, and quiet regions (Mathys and Solanki, 1989).

To estimate the errors in the deduced magnetic field strength, $B$, and surface filling factor, $f$, caused by this oversimplification, we construct model stellar atmospheres consisting of two components, quiet and flux-tube, based on solar models. We then synthesize, in LTE, Zeeman-sensitive and insensitive profiles ( $\lambda 8468$ and $\lambda 7748$ ) from this two-component atmosphere, and "analyze" them for Zeeman broadening using only a one-component quiet atmosphere.

\section{Model stellar flux-tube atmospheres}

We considered three models of the depth structure of magnetic flux tubes on active G2 stars, each based on solar models:

Tube A: $\quad$ HSRA evacuated by $50 \%, T(h)$ preserved, and Solanki (1986) solar flux tube used for $\log \tau_{\text {s000 }}<-2$

Tube B: $\quad$ Walton (1987) solar active region model (P1)

Starspot: Maltby et al. (1986), Umbral Sunspot

For the three model atmospheres and the quiet HSRA, we calculated intensity profiles for two Fe I lines $\lambda 8468$ (Landé $g=2.5, \chi_{\mathrm{ex}}=2.2 \mathrm{eV}$ ) and for $\lambda 7748$ (Landé $g=1.1, \chi_{\mathbf{e x}}=2.9 \mathrm{eV}$ ). The $g f$ values were taken from Blackwell et al. (1982). Line transfer is done by numerically solving the equation of transfer for each Stokes parameter in the model atmosphere, thus accounting for the Zeeman 
effect (Basri and Marcy, 1988). The important result is that the intensity profile shapes and equivalent widths that emerge from each atmosphere are significantly different, as shown in Figure 1.

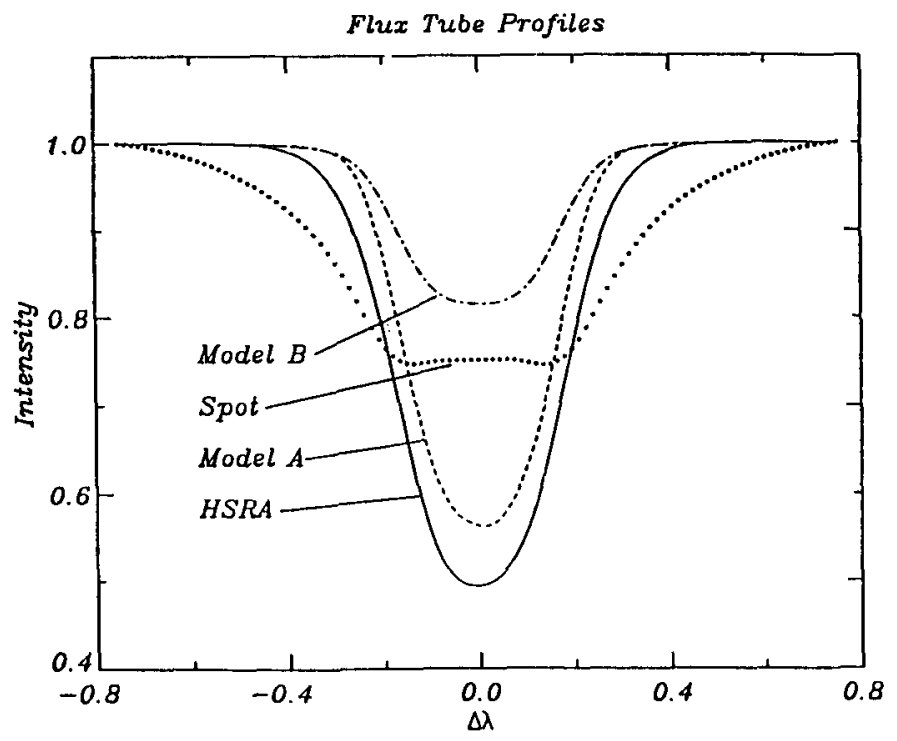

Fig. 1. Theoretical profiles for $\lambda 8468$ from four model atmospheres assuming $B=0 \mathrm{G}$ (but $B=2000 \mathrm{G}$ for spot).

\section{Two-component G-dwarf atmospheres}

For each theoretical two-component atmosphere, we construct disk-integrated profiles for $\lambda 8468$ and $\lambda 7748$ by adding the calculated flux profiles (weighted by continuum intensity) from the flux-tube and quiet regions in the proportion $30 \%: 70 \%$. Thus, the three atmospheres are:

1. $30 \%$ Tube A (1250 G) and $70 \%$ HSRA

2. $30 \%$ Tube $B(1250 \mathrm{G})$ and $70 \%$ HSRA

3. $30 \%$ Starspot $(2000 \mathrm{G})$ and $70 \%$ HSRA

The resulting composite profiles for $\lambda 8468$ and $\lambda 7748$ represent profiles that would be observed if magnetic regions on active G2 stars had atmospheres similar to solar flux tubes. However, in practice Zeeman broadening is determined without knowledge of flux-tube atmospheres; it is usually assumed that the atmosphere is quiet everywhere. Accordingly, we now determine $B$ and $f$ that would be derived from our "observations" of a two-component atmosphere if only a one-component HSRA atmosphere were used in the analysis. 


\section{One-component analysis}

Procedure:

1.Use HSRA $(B=0)$ to synthesize $\lambda 7748$ (Zeeman insensitive). Fit to "observed" two-component $\lambda 7748$. Free parameters are $[\mathrm{Fe} / \mathrm{H}]$ and macroturbulent velocity. 2.Using HSRA, $[\mathrm{Fe} / \mathrm{H}]$, and $V_{\text {mac }}$ from step 1 , synthesize $\lambda 8468$. Fit to "observed" two-component $\lambda 8468$. Free parameters are $B$ and $f$.

3.Repeat step 1 , but start with $B$ found in step 2 . Iterate.

The above procedure gives the values of $B$ and $f$ that would be obtained using a single quiet atmosphere to synthesize Zeeman-broadened profiles. Results are given in Table 1 . The above procedure was repeated, but the cores of $\lambda 8468$ were linearly scaled before fitting with $B$ and $f$, simulating the scaling procedure used by some investigators. Results are in the third section of Table 1.

Comments:

1. 50\% errors in $B$ and $f$ are made in a one-component analysis.

2.Model B (pure tube model) forces low $\mathrm{Fe} / \mathrm{H}$, due to line weakening.

3.Model A (evacuated plage) is milder: one-component analysis yields smaller errors.

4.Scaling cores slightly improves the performance of one-component analysis.

5.Errors in $B$ and $f$ are anti-correlated: $B f$ has smaller fractional error.

Table 1. Parameters required to achieve best fit to observations using a one-component atmosphere

\begin{tabular}{|c|c|c|c|c|c|c|c|c|c|}
\hline \multicolumn{3}{|c|}{ Input 2-Comp. } & \multicolumn{3}{|c|}{ 1-Comp. Fit } & \multicolumn{3}{|c|}{ Scaled 1-Comp. } & \multirow[b]{2}{*}[\mathrm{Fe}/\mathrm{H}]{} \\
\hline Model & $B$ & $f$ & $B$ & $f$ & $\sigma_{\text {fit }}$ & $B$ & $f$ & $\sigma_{\text {fit }}$ & \\
\hline Tube A & 1250 & $30 \%$ & 1500 & $27 \%$ & $0.77 \%$ & 1250 & $36 \%$ & $0.31 \%$ & -0.04 \\
\hline Tube $B$ & 1250 & $30 \%$ & 1900 & $16 \%$ & $0.48 \%$ & 1500 & $20 \%$ & $0.22 \%$ & -0.38 \\
\hline Starspot & 2000 & $30 \%$ & 2500 & $12 \%$ & $0.39 \%$ & 1000 & $9 \%$ & $0.39 \%$ & 0.00 \\
\hline
\end{tabular}

\section{Two-component K-dwarf atmospheres}

Crude two-component atmospheres have also been constructed for a hypothetical $\mathrm{K} 2$ dwarf, by scaling the solar flux-tube atmospheres. A Zeeman analysis of the calculated two-component profiles was done using only a quiet $\mathrm{K} 2$ dwarf atmosphere. Results are similar to those for G2 dwarf: We find significant errors in $B$ and $f,(\sim 50 \%)$ that are anti-correlated. The K-dwarf tube B model (30\% tube $\mathrm{B}+70 \%$ quiet) again yields very weak profiles, and in this case was not be fit by profiles from a quiet $\mathrm{K} 2$ atmosphere. 
Table 2. Parameters of best-fit Zeeman profile using quiet $\mathrm{K}$ atmosphere only

\begin{tabular}{|c|c|c|c|c|c|c|c|c|c|}
\hline \multicolumn{3}{|c|}{ Input 2-Comp. } & \multicolumn{3}{|c|}{ 1-Comp. Fit } & \multicolumn{3}{|c|}{ Scaled 1-Comp. } & \multirow[b]{2}{*}[\mathrm{Fe}/\mathrm{H}]{} \\
\hline Model & $B$ & $f$ & $B$ & $f$ & $\sigma_{\text {fit }}$ & $B$ & $f$ & $\sigma_{\text {fit }}$ & \\
\hline & 1250 & $30 \%$ & 1000 & $47 \%$ & $0.48 \%$ & 1000 & $53 \%$ & $0.49 \%$ & 0.00 \\
\hline Tube B & 1250 & $30 \%$ & & & & it atter & apted & & \\
\hline Starspot & 2000 & $30 \%$ & 2500 & $9 \%$ & $0.52 \%$ & 2000 & $12 \%$ & $0.41 \%$ & -0.06 \\
\hline
\end{tabular}

\section{Conclusions}

1. Systematic errors in $B$ and $f$ occur when line profiles from two-component stellar atmospheres are Zeeman-analyzed using one atmosphere.

2. Errors in $B$ and $f$ are anti-correlated; error in $B f$ is less than $20 \%$

3. Filling factors tend to be underestimated using a one-component atmosphere.

4. Two components are not enough to explain active stars.

a. Including flux-tubes in a quiet atmosphere yields lines weaker than observed.

b. Including flux-tubes also predicts active stars bluer than inactive stars not seen.

c. Luminosity would not be preserved, since tubes are twice as bright .

d. If flux-tubes are non-uniformly placed over surface, expect optical brightness variations of $\sim 1 / 2 \mathrm{mag}$ - not seen; observed mod. $\sim 0.02 \mathrm{mag}$

5. Active stars must have a third, cool atmospheric component that is spatially associated with flux-tubes to resolve issue 4.

\section{References}

Basri, G., Marcy, G.W.: 1988, Astrophys. J. 330, 274

Blackwell, D.E., Petford, A.D., Simmons, G.J.: 1982, Mon. Not. R. Astron. Soc. 210, 595

Maltby, P., Avrett, E.H., Carlsson, M., Kjeldseth-Moe, O., Kurucz, R.L., Loeser, R.: 1986, Astrophys. J. 306, 284

Marcy, G.W., Basri, G.: 1989, Astrophys. J. 345, 480

Mathys, G., Solanki, S.K.: 1989, Astron. Astrophys. 208, 189

Saar, S.: 1988, Astrophys. J. 324, 441

Solanki, S.K.: 1986, Astron. Astrophys. 168, 311

Solanki, S.K., 1987, in The Role of Fine-Scale Magnetic fields on the Structure of the Solar Atmosphere, ed. E.-H. Schroter, M. Vazquez, and A.A. Wyller (Cambridge University Press), p. 67

Walton, S.R., 1987, Astrophys. J. 312, 909 